\title{
CONTROL DE pH PARA PLANTA DE TRATAMIENTO DE AGUAS RESIDUALES
}

\author{
Wilson FABí́n AMAYA ${ }^{1}$, Óscar AlBerto CAÑón ${ }^{2}$, Óscar F. AvilÉs ${ }^{3}$ \\ (1)Ingeniero mecatrónico. leaper20@hotmail.com \\ (2)Ingeniero mecatrónico asesor de Proyectos Industriales FESTO Colombia. ocanon@festo.com.co \\ (3)Ingeniero eléctrico, especialista en Instrumentación Electrónica, profesor Universidad Militar Nueva Granada, estudiante \\ de maestría en sistemas automáticos de producción Universidad Tecnológica de Pereira. oaviles@umng.edu.co
}

Fecha de recepción:7 de junio de 2004

Fecha de aprobación: 7 de julio de 2004

Resumen-En este trabajo se presenta el problema de controlar el pH en una planta de tratamiento de aguas residuales (PTAR), la cual recibe soluciones ácidas provenientes de la producción de jugos (pH 2-5) que se dosifica con la sustancia usada para aseo (soda cáustica $0.04 \%$ ) con valores oscilatorios de pH 9-11. Se desarrolló e implementó un controlador clásico PI en un PLC comercial y se automatiza la PTAR mostrando el resultado obtenido en simulación en MatLAB.

Palabras clave: pH, PTAR, Controladores, PLC, SCADA.

\section{INTRODUCCIÓN}

$\mathrm{L}$ A Normatividad Internacional en Sistemas de Gestión Ambiental, se publicó en septiembre de 1996 por la Organización Internacional para la Normalización (con sede en Ginebra, Suiza). Allí se genera una norma efectiva en eco-gerencia (ISO 14000/14001 - sistemas de gestión ambiental) la cual se ha convertido en un requerimiento tanto para la competitividad como para la permanencia de empresas mediante factores relevantes para reducción de riesgos (ambientales y se puede enlazar a reducción en riesgos de seguridad y salud ocupacional).

El pH es una magnitud de mucha importancia en un sinnúmero de procesos biotecnológicos, como por ejemplo en la neutralización de desperdicios alimenticios. También ha cobrado gran relevancia en la minería y en el control de la contaminación, como es el caso de la neutralización de desechos industriales. El control de esta variable es en general difícil de realizar debido a la dependencia altamente no lineal entre los reactivos que ingresan al sistema y el $\mathrm{pH}$ que se establece. Esta no linealidad ya aparece en la definición, puesto que $p H=-\log \left(H^{+}\right)$.

Además, el control de $\mathrm{pH}$ en ocasiones presenta un comportamiento de fase no mínima [13]; de ahí que a veces al disminuir el flujo de algún reactivo, se produce una disminución de $\mathrm{pH}$ y en otras al aumentar este flujo se produce de igual modo una disminución de $\mathrm{pH}$.
Este trabajo muestra el control de una PTAR con entradas variables de $\mathrm{pH}$ causadas por la producción de jugos en su misma elaboración. Se procura mantener un $\mathrm{pH}$ de 8 a la entrada del floculador para optimizar el comportamiento del floculante y oxidante; esto es llevado a cabo con los mismos desperdicios de aseo de la planta, sin el uso de sustancias químicas adicionales, bajando el costo del proceso de purificación de agua.

El trabajo se divide en tres secciones: primero, se desarrolla el modelo del reactor, diseño del controlador y planta, con el fin de mostrar simulaciones en MatLAB y su respectiva implementación en el PLC ${ }^{1}$; en una segunda parte, se presenta el desarrollo del montaje eléctrico y mecánico; para finalizar, se presentan una serie de resultados en los cuales se pueden comparar los resultados de las simulaciones con la planta real, comportamiento de $\mathrm{pH}$ antes y después del controlador, el sistema de supervisión y márgenes de error.

Se utilizó un vástago estándar fabricado por la compañía AESCULAP, perteneciente al sistema de endoprótesis para la cadera BiCONTACT, con un cono de 12/14 mm., tamaño del vástago de $10 \mathrm{~mm}$., grosor sagital de $6.5 \mathrm{~mm}$., grosor distal de $7 \mathrm{~mm}$., largo del vástago de $150 \mathrm{~mm}$., y una cabeza ISODURf con cono 12/14 de $32 \mathrm{~mm}$. de diámetro. Este ha sido implantado en Colombia exitosamente con estudios de seguimiento por más de ocho años.

\section{MÉTODOS A.MODELO DEL PROCESO}

\section{Modelo del reactor}

Para realizar la modelación, se recurre a la ecuación del equilibrio de masa ${ }^{2}$ [12]; además, considerando el tanque de agitación continuo mostrado en la figura 1, se obtiene el balance másico representado en la ecuación 1 .

\footnotetext{
${ }^{1}$ PLC: Controladores Lógicos Programables.

${ }^{2}$ Razón de transferencia de masa de un elemento de fluido infinitesimal.
} 


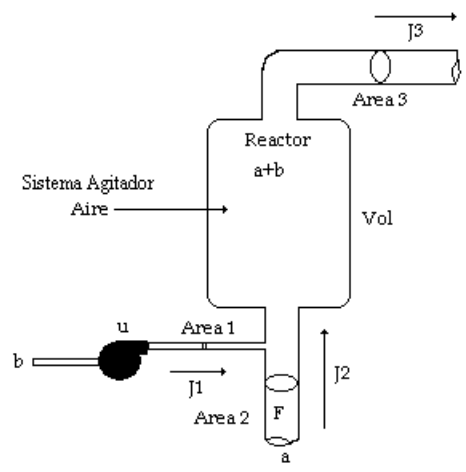

Figura 1. Esquema del reactor.

$$
r V=\frac{\partial y}{\partial t} V+J_{3} A_{3}-J_{1} A_{1}-J_{2} A_{2}
$$

Donde:

- $\quad r$ ) Tasa de remoción de primer orden. Por lo general esta tasa de remoción es generada por transferencia térmica, que en el caso de estudio es despreciable, ya que la pérdida de materia por transferencia de calor es insignificante en comparación con el flujo de transmisión. Por lo tanto $r=0$

- $\quad(V)$ Volumen del reactor (puede incluir tubería). [L]

- $\quad\left(J_{i}\right)$ Flujo másico. $\left[\mathrm{Kg} / \mathrm{m}^{2}{ }_{\mathrm{s}}\right]$

- $\quad\left(A_{i}\right)$ Área de tuberías. $\left[\mathrm{m}^{2}\right]$

- $(\partial y / \partial t)$ Es la variación de la concentración (Acumulación).[Kg/Ls]

Para que el sistema quede en función de las variables por manipular, se remplazan las unidades de la siguiente forma:

$$
\begin{aligned}
& J_{1} A_{1}=F * a \\
& J_{2} A_{2}=U * b \\
& J_{3} A_{3}=(F+U) * y
\end{aligned}
$$

Donde:

- $\quad(\boldsymbol{F})$ Caudal de la solución ácida (entrada de la planta).[L/s]

- (U) Caudal de la solución básica (variable manipulada).[L/s]

- (a ) Concentración de la sustancia ácida.[Kg./L]

- (b) Concentración de la sustancia básica.[Kg./L]

Desarrollando la operación se obtienen unidades consistentes:

$$
\begin{aligned}
J * A & =F^{*} a \\
\frac{K g}{m^{2} s} * m^{2} & =\frac{L}{s} * \frac{K g}{L} \\
\frac{K g}{s} & =\frac{K g}{s}
\end{aligned}
$$

Aplicando la transformada de Laplace a las ecuaciones (2), (3) y (4) y mediante manipulaciones algebraicas se obtiene:

$\frac{y}{a+\frac{U * b}{F}}=\frac{1}{\frac{V}{F} * s+1}$

que es la función de transferencia del sistema representado por el diagrama de bloques:

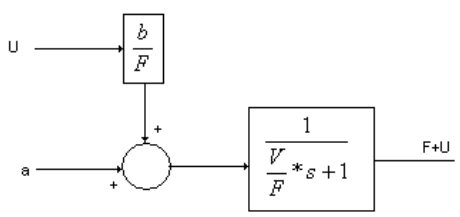

Figura. 2 Diagrama de bloques del sistema.

\section{Modelo químico de la rata de concentración}

Como $(y)$ es la concentración iónica, se recurre a la ecuación de equilibrio iónico ${ }^{3}[16]$ :

$y=\left|H^{+}\right|-\left|O H^{-}\right|$

Donde:

- $\mathrm{H}^{+}$iones de hidrógeno.

- $\mathrm{OH}^{-}$iones de hidróxido.

Esta ecuación representa la variación iónica entre la solución ácida y básica; para llevarlos a términos de $\mathrm{pH}$ se recurre a la ecuación ${ }^{4}$ [16]:

$p H=-\log \left(H^{+}\right)$

Despejando $H^{+}$se obtiene:

$H^{+}=10^{-p H}$

Ahora para remplazar el $\mathrm{OH}$ se recurre a la ecuación del producto iónico del agua ${ }^{5}[16]$ :

$\left\lfloor O H^{-}\right]\left[H^{+}\right\rfloor=K w$

Donde $K_{w}=10^{-14}$

Despejando $(\mathrm{OH})$ se obtiene:

$$
K w * 10^{p H}=\left|O H^{-}\right|
$$

Remplazando en la ecuación (8) y (10) en (6) se obtiene:

\footnotetext{
${ }^{3}$ Ecuación de equilibrio iónico. (El equilibrio iónico tiene que ver con la necesidad de una sustancia a presentar una carga neutra; estas cargas son producidas por los iones de hidrógeno generando así sustancias ácidas y básicas.)

${ }^{4}$ Ecuación de $\mathrm{pH}$. (Describe el comportamiento de acidez de una sustancia en términos de la concentración de iones de hidrógeno)

${ }^{5}$ Ecuación del producto iónico del agua.
} 
$y=10^{-p H}-10^{(p H-14)}$

que es la ecuación que representa la concentración en función de $\mathrm{pH}$.

\section{Linealización:}

Al analizar la respuesta dinámica de los procesos industriales, una de las mayores dificultades es su no linealidad, como es el caso del comportamiento de $\mathrm{pH}$ en el reactor. Para despejar el valor de pH de la ecuación (11), se recurre a linealizar [9] dicha expresión entre los valores de comportamiento más lineal, ubicados entre $(\mathrm{pH}=2-12)$; esto se muestra en la Figura 3. Evaluando en los valores estimados se obtiene:

$$
\begin{aligned}
& y(2)=0.01 \\
& y(12)=-0.01
\end{aligned}
$$

Se obtiene entonces la ecuación de la recta:

$$
p H=7-500 y
$$

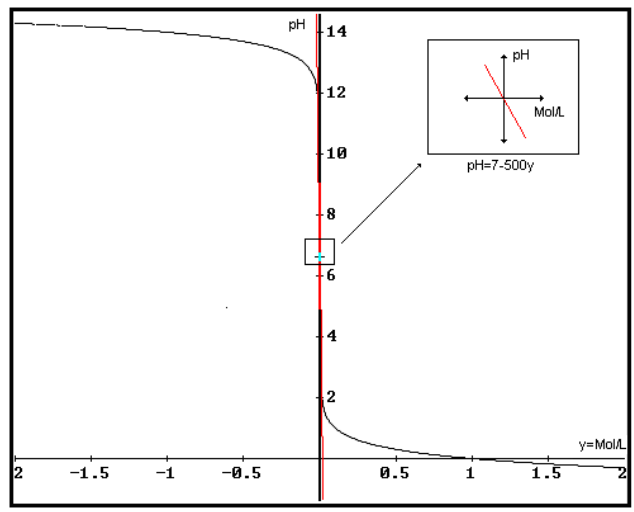

Fig 3 Gráfica de titulación del pH.

Con los anteriores resultados se obtiene el siguiente diagrama de bloques que representa el sistema, donde $f(u)$ es la ecuación (13).

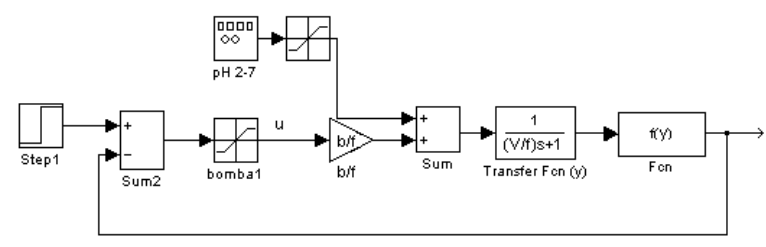

Fig. 4 Diagrama de bloques implementado en simulink.

En este modelo de la planta, la representación de la bomba es un elemento no lineal de memoria, el cual presenta un límite inferior y un límite superior que corresponden a los valores mínimos y máximos de caudal que puede proporcionar la bomba. Entre los dos límites se considera el comportamiento lineal. Tanto la bomba como el sensor no tienen una función de transferencia que los describa, ya que se considera el tiempo de respuesta mucho menor que el comportamiento de la planta, y no representan una variación significativa al modelo de la planta. Esto supone como resultado que $H(s)=1$.

La linealización se tuvo en cuenta para obtener las constantes del control PI. Para la implementación de los modelos en Simulink, se consideró la fórmula típica para reacciones entre bases fuertes y ácidos fuertes ${ }^{6}[20]$ :

$p H=-\log _{10}\left\lfloor\sqrt{0.25 y^{2}+10^{-14}}+0.5 y\right\rfloor$

\section{B. CONTROLADOR CLÁSICO PI DE LA PLANTA}

Se lleva a acabo un controlador clásico PI, típico para plantas de primer orden como el obtenido en el modelo; con esto se compensa la variación de la salida del sistema con respecto a una entrada de perturbación presente como lo es una solución ácida proveniente de los desperdicios de la producción de pulpas.

Función de transferencia controlador PI:

$D(s)=\frac{K_{p} s+K_{i}}{s}$

Para el diseño del controlador es necesario encontrar el Polinomio característico B(s), obtenido a partir de la función de transferencia del sistema, obteniendo de esta forma la expresión:

$$
\frac{\operatorname{out}(p H)}{\operatorname{In}(p H)}=\frac{\frac{-500}{V}\left(K_{p} s+K_{i}\right) b}{s^{2}+\left(\frac{F}{V}-\frac{500}{V} b K_{p}\right) s-\frac{500}{V} K_{i} b}
$$

Para determinar el comportamiento de la planta, se trabaja con el denominador de la función de transferencia, que es el polinomio característico:

$B(s)=s^{2}+\left(\frac{F-500 * b^{*} K_{p}}{V}\right) s-\frac{500}{V} K_{i} b$

Teniendo en claro esto se aplica el criterio de $\mathrm{ROUTH}^{7}$ para obtener los valores mínimos posibles que debe tomar las constantes $K_{p}$ y $K_{i}$ para mantener la estabilidad del sistema. Estas condiciones de estabilidad generaron los valores críticos de las constantes:

Para la constante proporcional se obtiene:

(Recordar que (b) es negativ;, por lo tanto es consistente la dirección del mayor)

\footnotetext{
${ }^{6}$ Ecuación que representa la variación de $\mathrm{pH}$ en función de la concentración para bases y ácidos fuertes.

${ }^{7}$ Método propuesto por Routh en 1874 con el cual se puede determinar el número de raíces de un polinomio con parte real positiva sin necesidad de encontrar realmente las raíces por métodos iterativos.
} 


$$
\begin{aligned}
& \frac{F-500 * b^{*} K_{p}}{V}>0 \\
& K_{p}>\frac{F}{500 * b}
\end{aligned}
$$

Para la constante integral se obtiene:

$$
\begin{aligned}
& -500 * b * K_{i}>0 \\
& K_{i}>0
\end{aligned}
$$

Para el diseño del controlador se maneja el método de igualación de polinomios el cual da la flexibilidad y confiabilidad para ver el comportamiento de la señal de salida con respecto a las necesidades de la planta; se supone un polinomio característico de las condiciones deseadas determinado por:

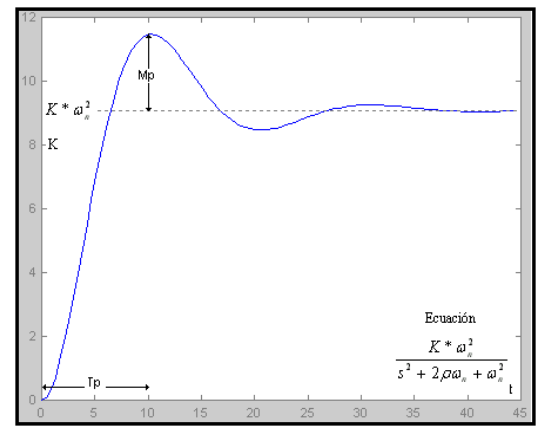

Fig. 5 Respuesta de segundo orden deseada para el sistema; gráfica realizada en MatLAB.

- $T_{p}=10 \mathrm{seg}$

- $M_{p}=2.2$

Se obtiene el polinomio característico deseado.

$B_{\text {ideal }}(s)=s^{2}+0.258197 s+0.115362$

La reacción química de la neutralización ocurre instantáneamente, pero el tiempo de mezcla física o real está más relacionado con la rata de dispersión y mezcla del reactor escogido. En muchos sistemas de neutralización se ha calculado un tiempo de $1 \frac{1}{2}$ a 30 minutos como un tiempo de retención mínimo para que ocurra la neutralización [15]. Teniendo en cuenta esto y el caudal de la sustancia ácida $(F)$ se puede obtener el volumen del reactor. Para el sistema implementado se aproximó un tiempo de 100 segundos ya que el caudal de la PTAR es demasiado alto y no existen retenedores antes del reactor.

$($ Tiempo $) *($ caudal $)=$ Volumen

$100 \mathrm{~s} * 10 \mathrm{~L} / \mathrm{s}=1000 \mathrm{~L}$
Para obtener los valores de las constantes del controlador, se remplazan los valores obtenidos en el modelo de control, en la ecuación (21)

- ( b ) Concentración de la sustancia básica. (-0.01 Kg./L)

- ( V ) Volumen (1000L)

- ( F ) Caudal de la solución ácida (entrada de la planta).[10 $\mathrm{L} / \mathrm{s}$ ]

Después de realizar las operaciones se obtienen las constantes para el sistema que logran el comportamiento deseado representado en la Figura 6:

- $K_{p}=50.1623$

- $K_{i}=23.14$

Se obtiene el siguiente resultado:

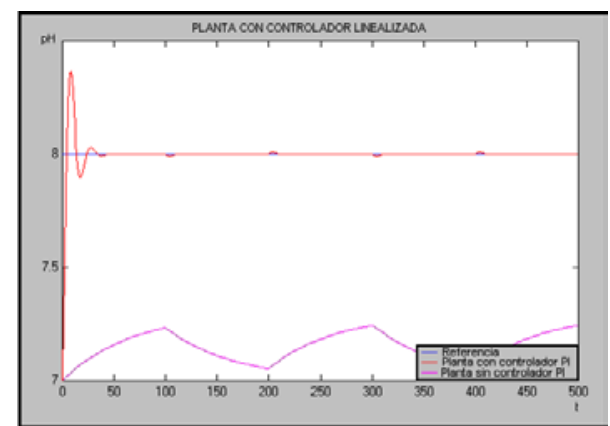

Figura. 6 Salida de la función de transferencia de la figura 3-2 (lineal izada) con y sin el controlador.

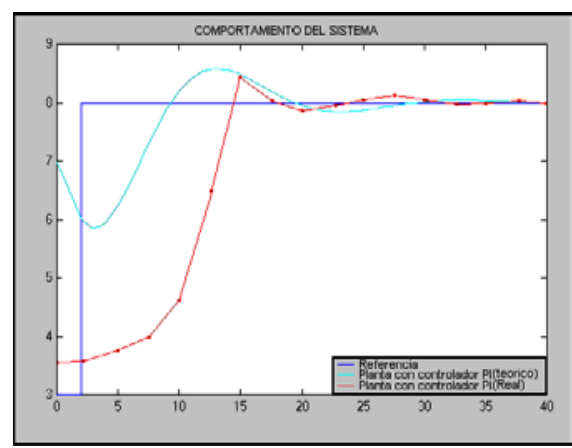

Figura. 7 Comportamiento del sistema real y teórico para un reactor de $1000 \mathrm{~L}$, un caudal de $8 \mathrm{~L} / \mathrm{s}$, y un $\mathrm{pH}$ de 3 .

\section{IMPLEMENTACIÓN Y RESULTADOS}

A continuación se muestra un bosquejo de la planta: 

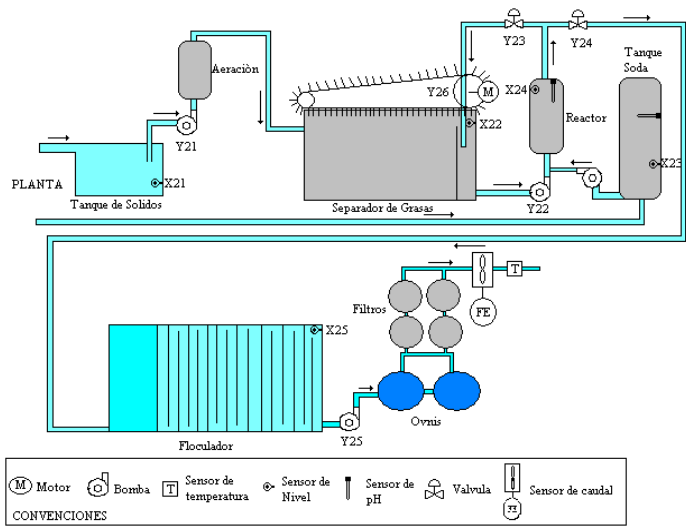

Figura. 8 Esquema general de la PTAR; con sus respectivas convenciones para el PLC.

Definido el controlador, se lleva a cabo la implementación del tablero de control; este presenta 2 variadores de velocidad Altivar 66, un PLC NAIS, una fuente Simens de 5 A, y sus respectivos contactores y trasmisores de $\mathrm{pH}$ (Hanna).
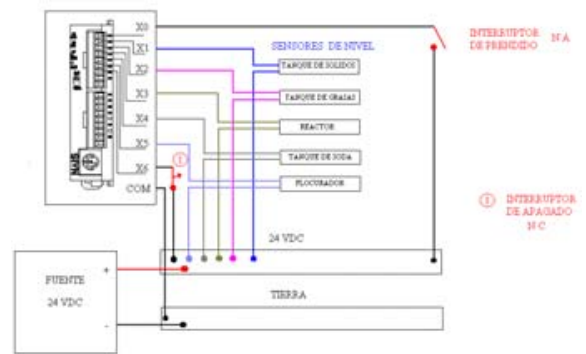

Figura. 9. Entradas digitales del PLC

Se puede ver en la Figura 9 que la alimentación de los sensores y pulsadores se llevó a cabo con una fuente de voltaje de 24VDC; presenta 5 sensores de nivel además de dos pulsadores, uno normalmente cerrado para el apagado, y el otro normalmente abierto para el encendido.

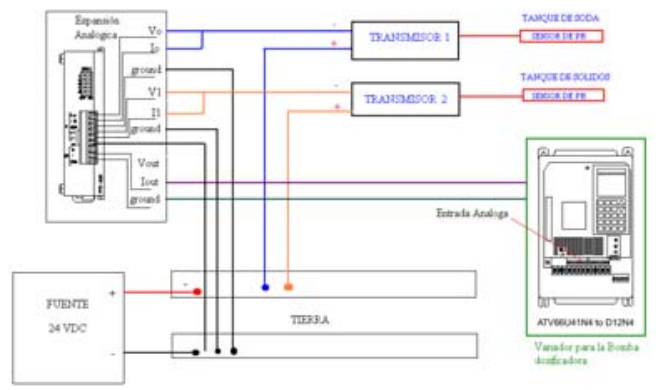

Figura. 10 Entradas y salidas análogas del PLC

En la Figura 10 se muestran las entradas (4-20mA) provenientes de los trasmisores de $\mathrm{pH}$ y salidas análogas (0-20mA) que van al variador Altivar 66 de la bomba dosificadora; este realiza la conversión a frecuencia y mueve el motor según su curva de funcionamiento.

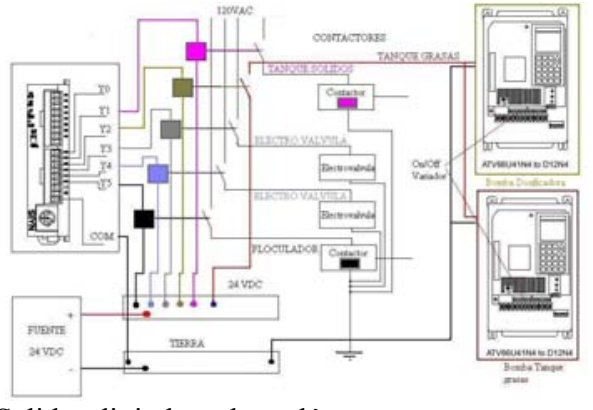

Figura.11. Salidas digitales a los relés, contactores.

En la Figura 10 se representa el circuito de salida, se ven 5 relés, dos de los cuales activan contactores para trifásica de los motores del floculador, y tanque de sólidos; dos salidas directas de 120VAC para las electroválvulas y una salida de 24VDC para el prendido y apagado de los variadores de la bomba dosificadora .

Por último, se desarrolló un programa de monitoreo, el cual utiliza Excel como soporte para almacenar datos.

Este programa consta de 2 ventanas principales: en la primera se ve el estado del controlador, bombas, nivel de tanques y electro válvulas, La segunda hoja presenta los datos registrados de $\mathrm{pH}$ en el reactor y en el tanque de sodas; este registro se presenta con fecha y hora. Para finalizar el programa prende una alarma sonora cuando el valor de $\mathrm{pH}$ en el tanque de soda es menor de 10, ya que con este valor la reacción química no alcanza a neutralizar un ácido de $\mathrm{pH} 3$ (pH mínimo proveniente de la producción de jugos). Una vez establecido el sistema, se monitoreó el pH a la entrada del floculador, punto en el cual se desea un $\mathrm{pH}$ de 8.5 para que al adicionarle oxidantes y coagulantes logre un valor de 7 , que es el $\mathrm{pH}$ ideal para el vertimiento del agua después del tratamiento. Tomando registros antiguos de $\mathrm{pH}$ y comparando los valores obtenidos al implementar el controlador, se puede ver en la Figura 12 que el $\mathrm{pH}$ toma valores estables y dentro de los valores deseados para el sistema; además se puede observar la dificultad de sostener el valor de $\mathrm{pH}$ en un valor exacto, causa de la variación molar de la sustancias dosificadora (Soda al $0.04 \%$ ) que proviene de aseos de la misma empresa.

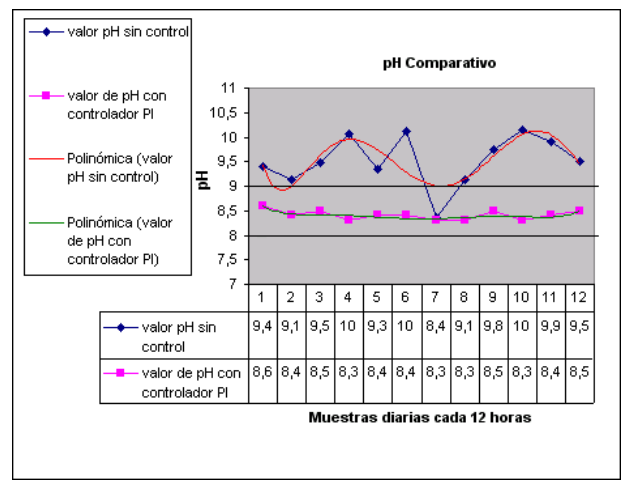

Fig 12. Figura comparativo de los valores de $\mathrm{pH}$ a la entrada del floculador antes y después del controlador. 
El comportamiento logrado con el controlador es estable y mejora los registros con la dosificación manual; se habla de un error de 2.3\%; mientras que el error obtenido anteriormente era de $15.3 \%$. Además, la recirculación garantiza que la sustancia neutralizada nunca entrará al floculador con un valor no deseado. Se tomaron varios tiempos de recirculación y, para un $\mathrm{pH}$ de 3 , el sistema tarda 12 minutos en neutralizarse; pero cuando el $\mathrm{pH}$ de entrada es de 6.5 , el sistema no presenta recirculación y es continua la dosificación de base.

\section{Conclusiones}

Después de haber analizado el problema, se concluyó que un controlador PI clásico era la mejor solución, ya que el sistema presentaba un comportamiento deseable; pese a las condiciones logarítmicas del $\mathrm{pH}$, se desarrollaron varias simulaciones donde se implementó el modelo dinámico, obtenido tras algunas deducciones y aproximaciones en MatLAB, logrando resultados del comportamiento de la planta, valores deseables del reactor, caudales, y determinando de esta forma las bombas por comprar. Estas simulaciones dan una idea muy clara acerca del comportamiento de la planta y las variables que la alteran, además de poder estudiar el sistema sin la necesidad de estar en el lugar de montaje.

Al realizar un estudio detallado de la planta se pudo generar un modelo flexible para algún cambio que se necesite realizar, además de poder funcionar en cualquier otra PTAR, o sistema que requiera neutralización de $\mathrm{pH}$, pudiendo encontrar las constantes $K_{i}$ y $K_{p}$ para diversos caudales, reactores y valores de concentración ácida y básica. La implementación se desarrolló en PLC ya que este ofrecía una mejor confiabilidad y cumplía con los estándares de seguridad internacionales. Los PLC cuentan con un sistema de supervisión, el cual guarda y muestra las gráficas en Excel para un buen monitoreo de $\mathrm{pH}$.

Se implementó una recirculación para evitar que el pH bajo de norma no pase al floculador. De esta forma no se vuelve tan critico el no usar sustancia dosificadora impura, sino que por el contrario permite usar los desperdicios de aseo de la planta, cuya concentración molar está en $0.04 \%$.

\section{REFERENCIAS}

[1] Ingeniería de control moderna. Ogata, editorial Prentice Hall.

[2] Control automático de procesos (Teoría y practica), Smith / Corripio, editorial Limusa.

[3] Control de sistemas dinámicos con retroalimentación. Franklin /Powell /Emami-Naeini; editorial Addison-Wesley Iberoamericana.

[4] Sistema de control en tiempo discreto. Ogata, editorial Prentice Hall.

[5] Tratamiento digital de señales. Proakis /Manolakis, editorial Prentice Hall.

[6] Dinámica de sistemas. Ogata, editorial Prentice Hall.
[7] Labview 6i, Antonio Manuel Lázaro, editorial Paraninfo. [8] Solución de problemas de ingeniería con matlab, Etter, editorial Prentice Hall.

[9] Matemáticas avanzadas para ingeniería vol. 2, Kreyszig, editorial Limusa.

[10] Sensores y acondicionadores de señal. Pamon Pallas Areny, editorial Marcombo.

[11] Electrónica de potencia (circuitos, dispositivos y aplicaciones). Rashid, editorial Prentice Hall.

[12] Mecánica de fluidos, Potter /Wiggert, editorial Prentice Hall.

[13] Tratamiento de aguas residuales (teoría y principios de diseño), Jairo Alberto Romero Rojas, editorial Escuela de Ingeniería.

[14] Ingeniería económica. Guillermo Baca, editorial Fondo Educativo Panamericano.

[15] Kart Ockert. "Practical Wastewater Pretreatment Strategies for Small Breweries" Publication no. T-2002-0.307-07 en la MBAA (master Brewers Association of the Americas).

[16] Bioquímica de Harper / Robert k. Murria, editorial El Manual Moderno.

[17] "pH control Systems", pH technical Article-PH30-DD.u. [18] "P2 Facts: pH Control", The Narragansett Bay Commission.

[19] “pH control”, HR 084053U003, IssuB 1.

[20] “Ácidos y Bases Duros y Blandos, Primera Parte, Principios Fundamentales", Ralph G. Pearson.

[21] "Automating Manufacturing Systems with PLC's", Hugh Jack, (Version 4.0, Mar31, 2002)

[22] "Autómatas programables" Josep Balcells /José Luis Romeral, Serie Mundo Electrónico, Marcombo editores.

[23] Ingenieria ambiental (contaminación y tratamientos). Ramón Sans Fonfria, editorial Alfaomega.

[24] Manual de NETZCH, Bombas NEMO.

[25] Instruction Manual HI8614, $\mathrm{pH}$ and ORP Transmitters, Hanna Instruments.

[26] Manual SIMENS SITOP power 5.

[27] ALTIVAR 66(Speed controllers for asynchronous motors) Programing manual. Telemécanique.

[28] Programable controller FP0, Hardware Manual.

[29] Manual de programación (para autómatas programables NAIS

[30] Análisis y Administración financiera. Gladys Carrillo de Rojas, cuarta edición. 